\title{
Doença celíaca associada à tireoidite de Hashimoto e síndrome de Noonan
}

\author{
Celiac disease associated with Hashimoto's thyroiditis and Noonan syndrome
}

Mariana Ortega Perez ${ }^{1}$, Giuliano Serafino Ciambelli ${ }^{1}$, Alcinda Aranha Nigri², Marta Wey Vieira ${ }^{3}$, Clóvis Duarte Costa (in memorian) ${ }^{4}$

\section{RESUMO}

Objetivo: Relatar o caso clínico de uma criança portadora de doença celíaca, tireoidite de Hashimoto e síndrome de Noonan.

Descrição do caso: Menina de dez anos e seis meses, branca, apresentando história de diarreia líquida há cinco meses e "aumento da barriga". Ao exame, mostrava peso de $20.580 \mathrm{~g}(\mathrm{P}<3)$, estatura de $114 \mathrm{~cm}(\mathrm{P}<3)$, hidratada, descorada $2+/ 4+$ e consciente. Presença de fácies triangular, com hipertelorismo ocular aparente, posição antimongoloide das fendas palpebrais, orelhas em abano de baixa implantação, micrognatia, pescoço curto e pectus excavatum. O abdome mostrava-se globoso, flácido, indolor, com hérnia umbilical, fígado a $2 \mathrm{~cm}$ do rebordo costal direito, linfedema em membro superior direito e edema de membros inferiores. Nos exames subsidiários, havia anemia microcítica e hipocrômica, déficit de proteínas totais, tireoidite de Hashimoto e atraso de cinco anos na idade óssea. Na ultrassonografia abdominal, as alças intestinais estavam levemente dilatadas. Devido ao linfedema e à diarreia crônica, a hipótese inicial foi de linfangiectasia intestinal, confirmada pela biópsia jejunal, que ainda mostrou padrão compatível de doença celíaca. O cariótipo foi $46 \mathrm{XX}$ com diagnóstico clínico de síndrome de Noonan.

Comentários: As doenças autoimunes se associam; no caso apresentado, a doença celíaca se associou à tireoidite de Hashimoto, possivelmente pela presença de antígenos do sistema HLA. Já a associação de doença celíaca à síndrome de Noonan é muito rara, sendo este o terceiro relato na literatura.
Palavras-chave: doença celíaca; síndrome de Noonan; doença de Hashimoto; linfangiectasia.

\section{ABSTRACT}

Objective: To describe the clinical case of a child with celiac disease, Hashimoto's thyroiditis and Noonan syndrome.

Case description: A Caucasian girl aged ten years and six months had liquid diarrhea for five months, and a "distended belly". At the physical exam: weight of $20,580 \mathrm{~g}$ $(\mathrm{P}<3)$, length of $114 \mathrm{~cm}(\mathrm{P}<3)$, hydrated, anemic $2+/ 4+$ and conscious. The patient presented triangular facies, apparent ocular hypertelorism, antimongoloid position of the palpebral fissures, ears with low implantation, micrognathia, short neck and pectus excavatum. The abdomen was globular, flaccid and painless; the liver was $2 \mathrm{~cm}$ below the right costal margin. Lymphedema in right upper limb and lower limb edema was also noted. Laboratory exams showed microcytic and hypochromic anemia, deficit of total proteins, Hashimoto's thyroiditis and a 5-year delay in bone age. Abdominal ultrasonography showed the bowel slightly dilated. Due to lymphedema and chronic diarrhea, the initial hypothesis was intestinal lymphangiectasis, which was confirmed by a jejunal biopsy, which also showed celiac disease. The genetic evaluation revealed a 46XX karyotype and a clinical diagnosis of Noonan syndrome.

Comments: Different autoimmune diseases can be associated. In this case, the celiac disease and the Hashimoto's thyroiditis are possibly related to the presence of HLA system antigens. However, the association of the celiac disease with
Endereço para correspondência:

Marta Wey Vieira

Rua Capitão Alfredo Cardoso, 226 - Jardim Faculdade

CEP: 18030-310 - Sorocaba/SP

E-mail: weyvieira@terra.com.br

Conflitos de interesses: nada a declarar

Recebido em: 15/7/2009

Aprovado em: 1/12/2009 
the Noonan syndrome is very rare, and this is the third report in the literature.

Key-words: celiac disease; Noonan syndrome; Hashimoto disease; lymphangiectasis.

\section{Introdução}

A doença celíaca, também denominada enteropatia glúten-sensível, é caracterizada por intolerância à prolamina, peptídeo que compõe o glúten. Em sua etiopatogenia estão envolvidos fatores ambientais, genéticos e imunológicos, configurando um padrão de vilosidades intestinais caracterizado por atrofia total ou subtotal, resultando na diminuição da absorção de nutrientes ${ }^{(1-4)}$.

A doença celíaca está associada a uma variedade de doenças desencadeadas por mecanismos autoimunes ou ligadas a antígenos do sistema HLA (antígeno leucocitário humano), importantes como coadjuvantes no seu diagnóstico ${ }^{(5)}$. Dentre essas afecções, destacam-se o diabetes melito tipo I e os distúrbios autoimunes da tireoide, entre eles a tireoidite de Hashimoto ${ }^{(1,6-8)}$.

A síndrome de Noonan é uma desordem de herança autossômica dominante que compreende baixa estatura, dismorfismos faciais, alterações torácicas e malformações cardíacas $^{(9-14)}$. Em 2001, Tartaglia et al ${ }^{(13)}$ atribuíram a etiologia da síndrome de Noonan a mutações no gene PTPN11 (protein tyrosine phosphatase, non-receptor type, 11), localizado no lócus 12q24.1.

Embora tenha sido elucidada do ponto de vista molecular, o diagnóstico de síndrome de Noonan ainda é essencialmente clínico, havendo necessidade de investigar outras entidades que também cursam com baixa estatura, como é o caso da síndrome de Turner, da síndrome de Aarskog e da síndrome de Leopard $^{(8,10,15)}$.

O objetivo deste artigo foi relatar e discutir o caso clínico de uma criança portadora de doença celíaca, tireoidite de Hashimoto e síndrome de Noonan. Para tanto, foi obtido Termo de Consentimento Livre e Esclarecido e a aprovação pelo Comitê de Ética em Pesquisa da Pontifícia Universidade Católica de São Paulo.

\section{Descrição do caso}

Paciente do sexo feminino, com dez anos e seis meses de idade, branca, com queixa há cinco meses de diarreia líquida, fezes amareladas, fétidas e brilhantes, uma vez ao dia. Desde o nascimento, apresentava "aumento da barriga”, com dificuldade para eliminar gases. Paciente negou muco

Quadro 1- Exames subsidiários da paciente.

\begin{tabular}{|c|c|c|}
\hline Exames & \multicolumn{2}{|l|}{ Resultado } \\
\hline Eritrograma & \multicolumn{2}{|l|}{ Anemia microcítica e hipocrômica } \\
\hline \multirow{2}{*}{$\begin{array}{l}\text { Hormônios e autoanticorpos } \\
\text { tireoidianos }\end{array}$} & $\mathrm{TSH}=9,27 \mathrm{mUl} / \mathrm{mL}(\mathrm{VR} 0,5-5,1)$ & Antimicrossomal=1/400 (VR $\leq 1 / 100)$ \\
\hline & $\mathrm{T} 4=8,1 \mu \mathrm{g} / \mathrm{dL}(\mathrm{VR} 5-13)$ & Antitireoglobulina $=1 / 100(\mathrm{VR} \leq 1 / 100)$ \\
\hline Anti-ilhotas de Langerhans & \multicolumn{2}{|l|}{ Negativo } \\
\hline \multirow[t]{3}{*}{ Eletroforese de proteínas } & Proteínas totais: $3,5 \mathrm{~g} / \mathrm{dL}(\mathrm{VR} 6-8)$ & Fração $\alpha_{2}: 0,9 \mathrm{~g} / \mathrm{dL}($ VR 0,4-0,7) \\
\hline & Albumina: $1,3 \mathrm{~g} / \mathrm{dL}(\mathrm{VR} 3,5-5,0)$ & Fração $\beta: 0,6 \mathrm{~g} / \mathrm{dL}(\mathrm{VR} 0,7-0,9)$ \\
\hline & Fração $\alpha_{1}: 0,2 \mathrm{~g} / \mathrm{dL}(\mathrm{VR} 0,2-0,4)$ & Fração $\gamma: 0,4 \mathrm{~g} / \mathrm{dL}(\mathrm{VR} 1-1,7)$ \\
\hline \multirow[t]{3}{*}{ Dosagem de imunoglobulinas } & \multicolumn{2}{|l|}{ IgA: 63mg/dL (VR 60-300) } \\
\hline & \multicolumn{2}{|l|}{ IgG: 610mg/dL (VR 630-1400) } \\
\hline & \multicolumn{2}{|c|}{ IgM: $80 \mathrm{mg} / \mathrm{dL}(\mathrm{VR} 30-148)$} \\
\hline Ultrassonografia abdominal & \multicolumn{2}{|c|}{ Alças intestinais levemente dilatadas com peristaltismo e com líquido na luz } \\
\hline Biópsia intestinal & \multicolumn{2}{|c|}{$\begin{array}{l}\text { Fragmento de intestino delgado exibindo marcante atrofia vilositária (índice vilo/cripta } \\
\text { 1:10) com hiperplasia das células de revestimento das criptas, onde se observa } \\
\text { aumento da atividade mitótica. Presença de área focal com aumento do número de } \\
\text { linfócitos intraepiteliais, com relação linfócito intraepitelial/célula epitelial de } 1: 1 . \mathrm{Na} \\
\text { lâmina própria, ectasia de capilares linfáticos com alguns macrófagos xantomizados } \\
\text { de permeio }\end{array}$} \\
\hline Idade óssea & \multicolumn{2}{|c|}{ Atraso em 5 anos } \\
\hline Ecocardiograma & \multicolumn{2}{|c|}{ Normal, de acordo com o sexo e a idade } \\
\hline
\end{tabular}

VR: valor de referência 
e sangue nas fezes, febre e vômitos. Nasceu a termo, parto normal, pais não-consanguíneos, com $2750 \mathrm{~g}$, comprimento de $49 \mathrm{~cm}$, perímetro cefálico de $34 \mathrm{~cm}$ e torácico de $32 \mathrm{~cm}$, apresentando linfedema em membro superior direito. Houve referência a um irmão prematuro, falecido com um mês de vida por leucemia. A alimentação era composta por dieta geral e o desenvolvimento neuropsicomotor estava adequado para a idade cronológica.

Ao exame físico, encontrava-se em bom estado geral, mucosas descoradas $2+/ 4+$ e hidratadas, tecido celular subcutâneo preservado. O peso era $20580 \mathrm{~g}$ (percentil-P<3), a estatura $114 \mathrm{~cm}(\mathrm{P}<3)$, o índice de massa corpórea (IMC) era $15,83 \mathrm{~kg} / \mathrm{m}^{2}(25<\mathrm{P}<0)$ e a pressão arterial, $105 \times 60 \mathrm{mmHg}$.

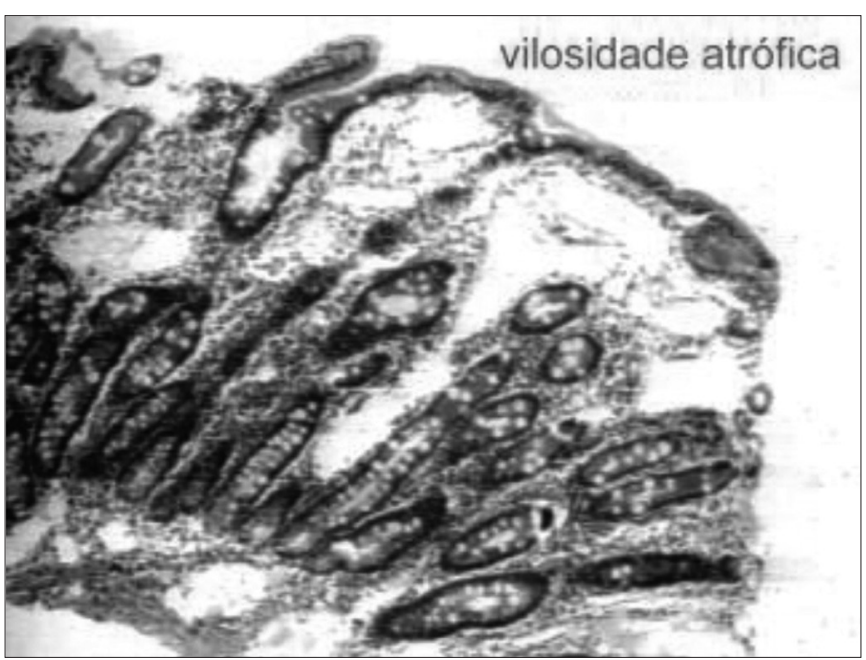

Figura 1 - Mucosa do intestino delgado exibindo marcante atrofia vilositária, com hiperplasia das células de revestimento das criptas (HE 40x).

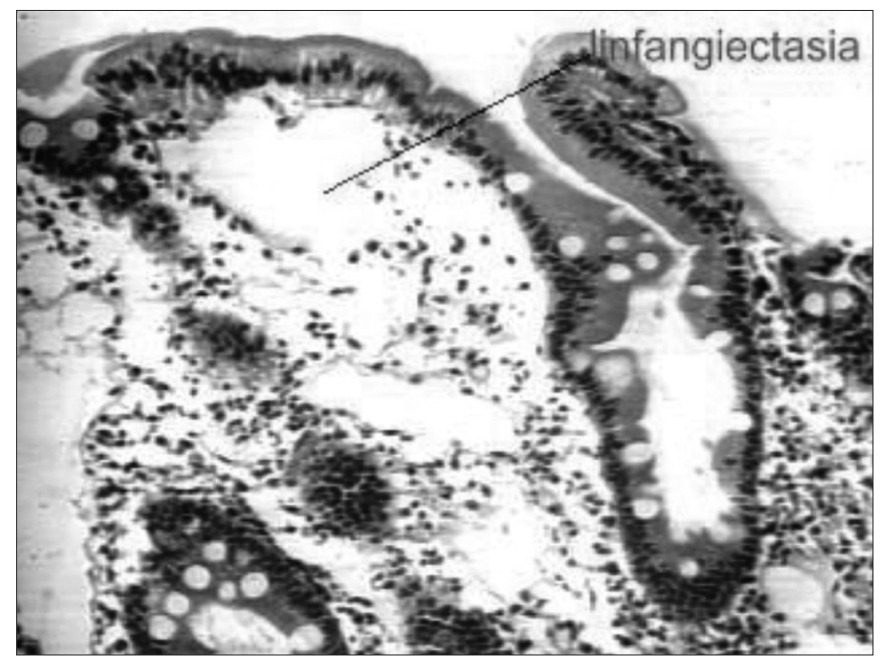

Figura 2 - Mucosa do intestino delgado evidenciando área de ectasia de capilares linfáticos em lâmina própria (HE 100x).
Notou-se a presença de fácies triangular e hipertelorismo ocular aparente, com distância intercantal externa de $9,0 \mathrm{~cm}$ $(75<\mathrm{P}<97)$, distância intercantal interna de $3,0 \mathrm{~cm}(\mathrm{P}=50)$ e índice cantal de 33 (limite superior de normalidade $=38$ ). Havia posição antimongoloide das fendas palpebrais, orelhas em abano de baixa implantação, palato arqueado, micrognatia, pescoço curto e pectus excavatum. Não foram notadas alterações nos aparelhos respiratório e cardiovascular. O abdome era globoso, flácido, com diástase de músculo reto abdominal, hérnia umbilical, fígado a $2 \mathrm{~cm}$ da reborda costal direita e baço não-palpável. Notou-se edema $+/ 4+$ em membros inferiores, perfusão periférica inalterada, edema acentuado em membro superior direito e unhas quebradiças em mãos e pés. Genital feminino sem alterações, desenvolvimento pré-puberal (M1 P1 pelo estadiamento de Tanner). O Quadro 1 traz os exames subsidiários realizados pela paciente.

$\mathrm{Na}$ evolução, o TSH mostrou-se aumentado e o anticorpo antimicrossomal positivo, sugerindo tireoidite crônica de Hashimoto, iniciando-se o tratamento com hormônio tireoidiano. Posteriormente, foi realizada a biópsia de intestino delgado, em que foram obtidos quatro fragmentos que exibiram marcante atrofia vilositária (índice vilo/cripta 1:10), com hiperplasia das células de revestimento das criptas, nas quais havia aumento da atividade mitótica (Figura 1). Observouse ainda área focal com aumento do número de linfócitos intraepiteliais, com relação linfócito intraepitelial/célula epitelial de 1:1. Na lâmina própria, havia ectasia de capilares linfáticos (Figura 2), com alguns macrófagos xantomizados de permeio. Tais achados sugeriram o diagnóstico de doença celíaca e linfangiectasia intestinal. Frente ao diagnóstico de doença celíaca, foi instituída dieta isenta de glúten.

À avaliação genética, o cariótipo resultou 46XX, chegando-se, ao final da avaliação clínica, à conclusão diagnóstica de síndrome de Noonan.

\section{Discussão}

A doença celíaca é uma enteropatia autoimune que se instala em indivíduos predispostos, em decorrência de uma intolerância à prolamina, peptídeo componente do glúten. Sua fisiopatologia não está completamente elucidada, porém acredita-se na participação de fatores genéticos, ambientais e imunológicos ${ }^{(1,2)}$.

Os determinantes genéticos são resultantes da presença de antígenos do sistema HLA que conferem suscetibilidade à doença celíaca, especialmente as formas HLA-DQ2 e HLADQ8 ${ }^{(2)}$. Somado a isso, existe ainda a influência ambiental 
- representada pela exposição ao glúten - além dos fatores imunológicos, que colocam em evidência os linfócitos CD4, bem como citocinas inflamatórias, capazes de alterar a mucosa intestinal, prejudicando a absorção ${ }^{(3,16)}$.

Frente ao distúrbio absortivo, a paciente em questão apresentou anemia ferropriva, baixa concentração de proteínas plasmáticas totais e idade óssea aquém do esperado, situação também atribuída à síndrome de Noonan. Em relação à deficiência proteica, além do comprometimento celíaco, a criança também apresentava linfangiectasia intestinal; ou seja, a paciente era portadora de duas enteropatias perdedoras de proteínas, justificando essa carência.

De maneira geral, o diagnóstico da doença celíaca é feito inicialmente por uma triagem, ocasião em que são dosados anticorpos, como antiendomísio e antitransglutaminase; contudo, a biópsia intestinal é o padrão-ouro no diagnóstico da doençç ${ }^{(17,18)}$. De acordo com os critérios da European Society of Paediatric Gastroenterology and Nutrition (ESPGAN) da década de 1970, revisados em 1990, o paciente com suspeita de doença celíaca pode ter o diagnóstico confirmado por biópsia única de intestino delgado, mostrando atrofia vilositária com hiperplasia das criptas e aumento dos linfócitos intraepiteliais. Paralelamente, a ESPGAN adota ainda como critério a remissão dos sintomas após a instituição de dieta isenta de glúten, que pode ocorrer, usualmente, em semanas a meses ${ }^{(17)}$. A paciente em questão foi diagnosticada diretamente pela biópsia, que trouxe achados típicos de doença celíaca, como atrofia vilositária, hiperplasia das células de revestimento das criptas e aumento do número de linfócitos intraepiteliais ${ }^{(18)}$. Diante do diagnóstico, foi instituída a dieta isenta de glúten e a paciente se mostrou assintomática após três meses. Convém ressaltar que a paciente foi seguida por dez anos no Ambulatório de Gastroenterologia Pediátrica da Faculdade de Medicina de Sorocaba da PUC-SP, mantendose assintomática após todos esses anos de dieta isenta de glúten. Vale lembrar que, no caso clínico relatado, não foi realizada dosagem de anticorpos, pois existia, inicialmente, a suspeita de linfangiectasia intestinal, haja vista a presença de linfedema em membro superior direito (Figura 3), associado ao quadro de diarreia crônica.

Mesmo a paciente não apresentando manifestação clínica de tireoidite de Hashimoto, seu déficit de crescimento, bem como o atraso importante da idade óssea (cinco anos) exigiram avaliação tireoidiana, que detectou positividade para anticorpo antimicrossomal, além de níveis aumentados de TSH. Nesse contexto, as doenças autoimunes somam-se, como é o caso da paciente em questão, que desenvolveu, paralelamente, doença celíaca e tireoidite de Hashimoto. Tal associação resulta da presença de fatores genéticos, especialmente antígenos do sistema HLA comuns tanto à doença celíaca como à tireoidite de Hashimoto, entre eles o HLA DQ2, o DQ8, o DR3 e o DR4 $4^{(2,19)}$. Hadithi et al $l^{(19)}$ apontaram que $5(4,8 \%)$ dos 104 pacientes holandeses com tireoidite de Hashimoto tinham doença celíaca, de acordo com os critérios da ESPGAN. Paralelamente, 22 (12\%) dos 184 celíacos incluídos no mesmo estudo apresentavam critérios clínicos e laboratoriais compatíveis com tireoidite de Hashimoto.

Considerando que uma doença autoimune pode estar associada ao surgimento de outra, convém realizar a triagem sorológica para doença celíaca em diabéticos do tipo 1, uma vez que a prevalência de doença celíaca é cerca de dez vezes maior nesses pacientes, em comparação à população geral ${ }^{(20)}$. O contrário também se aplica, ou seja, indica-se a realização

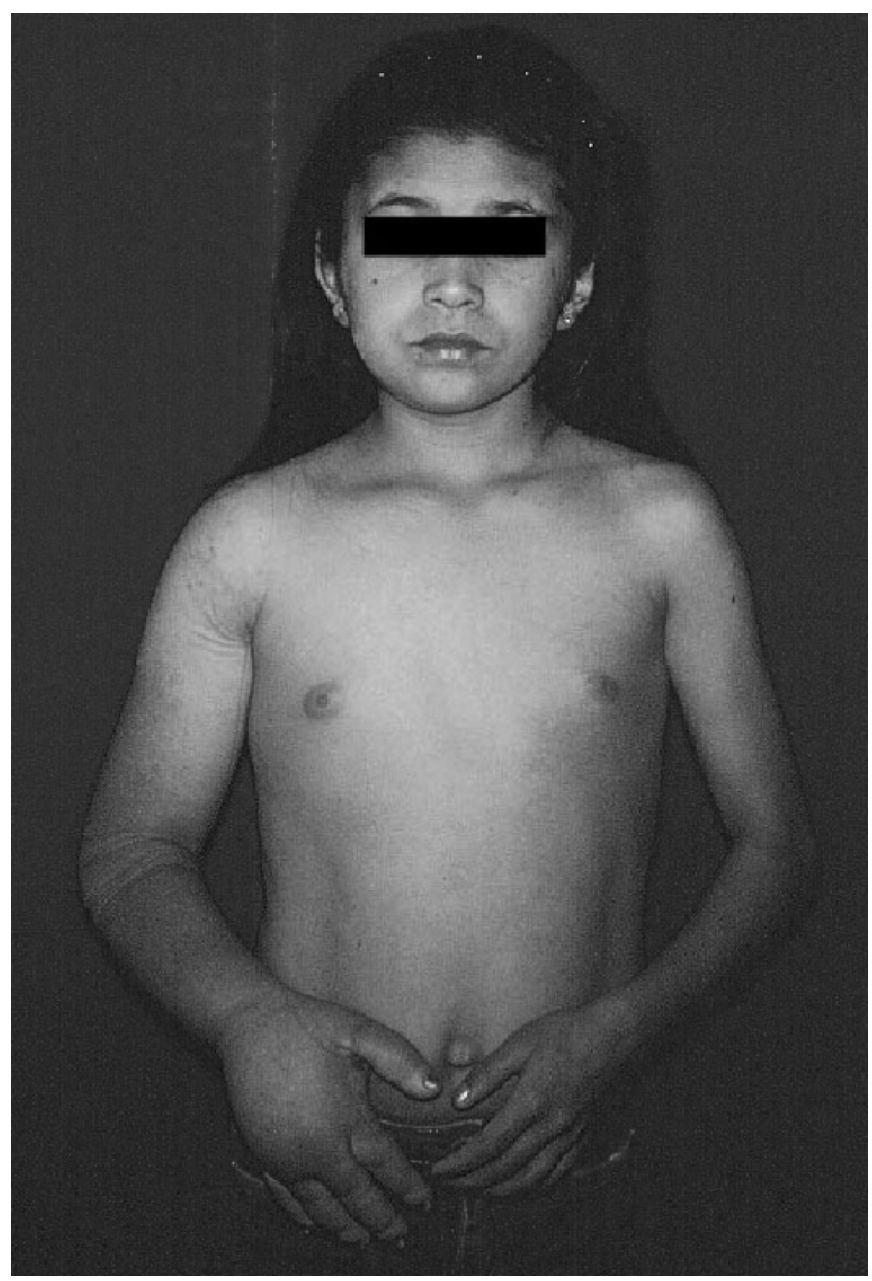

Figura 3 - Aspecto geral da paciente. Notar a presença de linfedema em membro superior direito. 
de triagem de diabetes melito tipo 1 em celíacos, como ocorreu no caso em questão, cujo resultado foi negativo para anticorpo anti-ilhotas de Langerhans.

É pouco usual a relação entre doença celíaca e síndrome de Noonan, sendo o presente relato o terceiro a apontar esse binômio. Amoroso et a $l^{(9)}$ foram os primeiros a documentar essa associação ao descrever uma paciente de 26 anos com lúpus eritematoso sistêmico e tireoidite de Hashimoto, além da síndrome de Noonan. No mesmo ano, Tagarro ${ }^{(11)}$ relatou o caso de uma criança diagnosticada com doença celíaca aos 20 meses e síndrome de Noonan aos quatro anos.

A síndrome de Noonan é uma desordem autossômica dominante caracterizada por uma variedade de fenótipos, incluindo baixa estatura, fácies dismórfica, malformações cardíacas e alterações torácicas ${ }^{(5,9-14)}$. Seu diagnóstico é difícil, especialmente em indivíduos sem alterações cardíacas ou em adultos, quando os dismorfismos faciais tornam-se menos perceptíveis( ${ }^{(5)}$. O diagnóstico diferencial de síndrome de Noonan com outras entidades clínicas é imprescindível, entre elas a síndrome de Turner, que engloba uma série de eventos fenotípicos que coincidem com os da síndrome de Noonan ${ }^{(5,10,11)}$. Nesse caso, o cariótipo da paciente resultou em 46XX, excluindo a síndrome de Turner. As demais hipóteses diagnósticas, entre elas a síndrome de Leopard e a síndrome de Aarskog, foram afastadas clinicamente ${ }^{(10)}$.

Embora a síndrome de Noonan tenha sido elucidada do ponto de vista molecular, o diagnóstico clínico ainda é usado para a confirmação diagnóstica ${ }^{(5,10)}$. Van der Burgt et al $l^{(15)}$ estabeleceram alguns critérios diagnósticos para a síndrome de Noonan (Quadro 2) na tentativa de objetivar sua investigação clínica. O diagnóstico da paciente foi concluído com dois critérios maiores (altura inferior ao percentil 3 e pectus excavatum) e dois critérios menores (face sugestiva e displasia linfática).

Convém ressaltar que, nos antecedentes familiares da paciente, há referência a um irmão que faleceu com um mês de vida em virtude de leucemia. Acredita-se na hipótese de que esse irmão pudesse ser portador da mesma mutação, o que configuraria um critério adicional no diagnóstico de síndrome de Noonan já realizado. No entanto, tal critério não foi contemplado para o diagnóstico clínico já que não havia meios para confirmação.

Tartaglia $e t a^{(14)}$, ao estudarem 62 crianças com leucemia mielomonocítica juvenil sem síndrome de Noonan, observaram que $34 \%$ delas possuíam mutação do PTPN11 no tecido hematopoiético. Segundo Watkins et al ${ }^{(21)}$, pacientes com leucemia mieloide aguda e síndromes mielodisplásicas também estão sujeitos a apresentar mutações no gene PTPN11, numa porcentagem de 14 e $10 \%$, respectivamente. Além disso, a atividade alterada da proteína SHP-2, em decorrência da mutação do PTPN11, aumenta a sinalização intracelular da via Ras, a qual desempenha papel importante na origem das doenças mieloproliferativas ${ }^{(5)}$.

No que diz respeito às anomalias do sistema linfático na síndrome de Noonan, estas surgem em cerca de $20 \%$ dos casos, sendo que o linfedema costuma persistir na idade adulta, ao contrário do que ocorre na síndrome de Turner. Além do linfedema, a paciente aqui relatada apresentou linfangiectasia intestinal, confirmada pela biópsia jejunal.

Quadro 2 - Critérios de van der Burgt et a/(21) para diagnóstico da síndrome de Noonan

\begin{tabular}{|lll|}
\hline & & Critérios \\
\cline { 2 - 3 } & Maiores & Menores \\
\hline Facies & Típica* & Sugestiva \\
Alterações cardíacas & Estenose valvar pulmonar & Outras \\
& Miocardiopatia hipertrófica & \\
Estatura & $<3^{\circ}$ percentil & $<10^{\circ}$ percentil \\
Alterações torácicas & Pectus carinatum e/ou excavatum & Alargado \\
História familiar & Parente de $1^{\circ}$ grau com diagnóstico & Parente de $1^{\circ}$ grau sugestivo de \\
& de síndrome de Noonan & síndrome de Noonan \\
Outros & Todos os abaixo: & Qualquer um de \\
& Retardo mental & Retardo mental \\
& Criptorquidia & Criptorquidia \\
& Displasia linfática & Displasia linfática \\
\hline
\end{tabular}

*Face triangular, fenda, palpebral oblíqua com o ângulo externo voltado para baixo, hipertelorismo ocular, ptose palpebral, pavilhão auricular malformado e de implantação baixa, micrognatia, pescoço alado. Diagnóstico de síndrome de Noonan: face típica mais um outro critério maior ou dois menores; face sugestiva mais dois outros critérios maiores ou três menores. 
A linfangiectasia intestinal pode ser secundária a uma série de doenças que ocasionam obstrução linfática, tais como fibrose retroperitoneal, pancreatite crônica, tumores abdominais ou retroperitoneais, tuberculose mesentérica, doença de Crohn, má rotação intestinal, doença de Whipple, pericardite constritiva, insuficiência cardíaca congestiva e doença celíaca ${ }^{(22,23)}$. Entretanto, também pode ter apresentação isolada ou ainda fazer parte do espectro de manifestações clínicas de outras doenças, como macroglobulinemia, infecções cutâneas virais, síndrome nefrótica, hipobeta-lipoproteinemia, linfomas e síndromes como Noonan, Klippel-Trénaunay-Weber, Turner, Hennekan e yellow nail(24). A biópsia constitui o padrão-ouro no diagnóstico da linfangiectasia intestinal, cujo principal estigma é a presença de ductos linfáticos dilatados na lâmina própria, mucosa e submucosa $a^{(22,24,25)}$. Pode haver ainda o alargamento e a distorção das vilosidades intestinais, sem sinais de atrofia vilositária e infiltração celular ${ }^{(23,26)}$.

Já as malformações cardíacas ocorrem em cerca de 50\% dos portadores de Noonan, destacando-se a estenose pulmonar valvar, a miocardiopatia hipertensiva, a comunicação interatrial, a comunicação interventricular, a tetralogia de Fallot e o prolapso da valva mitral ${ }^{(9-11)}$. Neste caso, a paciente realizou ecocardiograma que se apresentou sem alterações.

De todos os critérios de Burgt, um dos mais expressivos é a baixa estatura, sendo a síndrome de Noonan um importante diagnóstico diferencial em crianças com déficit de crescimento ${ }^{(5,10,15)}$. A baixa estatura da paciente pode ser atribuída tanto à síndrome de Noonan como à doença celíaca, esta última em virtude das perdas intestinais ${ }^{(3)}$. Além de favorecer o retorno do crescimento, a dieta isenta de glúten parece exercer influência sobre os autoanticorpos da tireoidite de Hashimoto. De acordo com Ventura et al ${ }^{(27)}$, os anticorpos antitireoide tendem a desaparecer após a introdução da dieta, assim como os anticorpos presentes na doença celíaca. $\mathrm{Na}$ paciente em questão, a dieta não exerceu influência sobre a normalização dos anticorpos antitireoidianos. O anticorpo antimicrossomal mostrou-se não-reagente antes da isenção do glúten e após um ano e três meses do tratamento do hipotireoidismo.

Desse modo, é imprescindível o rápido diagnóstico da doença celíaca com a retirada do glúten da dieta, na tentativa de evitar o agravamento do quadro, bem como o desenvolvimento de outras doenças autoimunes, haja vista que celíacos não-tratados têm maior chance de apresentar outras doenças autoimunes ${ }^{(6)}$. Uma particularidade desse caso foi o diagnóstico tardio de síndrome de Noonan, que se deu aos 14 anos e oito meses, o que serve de alerta para os pediatras, por se tratar de uma síndrome que pode cursar com dismorfismos sutis, sendo predominante o retardo pôndero-estatural. Finalizando, a associação entre doença celíaca e síndrome de Noonan é rara, sendo este o terceiro relato na literatura e o primeiro no Brasil.

\section{Agradecimentos}

Agradecemos à professora Doutora Dorina Barbieri, que gentilmente analisou este trabalho, trazendo grandes contribuições; à professora Doutora Maria Cecília Ferro e ao professor doutor Nelson Brancaccio dos Santos, que contribuíram para o estudo anatomopatológico; à senhora Elizabeth Almeida, pelo preparo das lâminas. Em especial, agradecemos ao professor Doutor Clóvis Duarte Costa, in memorian, fundamental neste trabalho.

\section{Referências bibliográficas}

1. Costa CD. Condições associadas à doença celíaca. J Bras Med 2003; 85:41-3.

2. Craig S, Robins G, Howdle PD. Advances in celiac disease. Curr Opin Gastroenterol 2007;23:142-8

3. Gueiros AC. Doença celíaca como causa de baixa estatura em crianças e adolescentes [dissertação de mestrado]. Recife (PE): UFPE; 2005.

4. Shaoul R, Lerner A. Associated autoantibodies in celiac disease. Autoimmun Rev 2007;6:559-65.

5. Ferreira LV, Souza SA, Montenegro LR, Arnhold IJ, Pasqualini T, Heinrich $\mathrm{JJ}$ et al. Phenotype variability in Noonan syndrome patients with and without PTPN11 mutation. Arq Bras Endocrinol Metabol 2007;51:450-6.

6. Brandt KG, Silva GA, Antunes MM. Doença celíaca em um grupo de crianças e adolescentes portadores de diabetes mellitus tipo I. Arq Bras Endocrinol Metabol 2004;48:823-7.

7. Ch'ng CL, Jones MK, Kingham JG. Celiac disease and autoimmune thyroid disease. Clin Med Res 2007;5:184-92.

8. Iuorio R, Mercuri V, Barbarulo F, D'Amico T, Mecca N, Bassotti G et al. Prevalence of celiac disease in patients with autoimmune thyroiditis. Minerva Endocrinol 2007;32:239-43.

9. Amoroso A, Garzia P, Vadacca M, Galluzzo S, Del Porto F, Mitterhofer AP et al. The unusual association of three autoimmune diseases in a patient with Noonan syndrome. J Adolesc Health 2003;32:94-7.

10. Bertola DR. Estudo do gene PTPN11 nos pacientes afetados pela Síndrome de Noonan [tese de mestrado]. São Paulo (SP): USP; 2006.

11. Tagarro A. Celiac disease and Noonan syndrome. Pediatrika 2003;23:28-32

12. Tartaglia M, Martinelli S, Stella L, Bocchinfuso G, Flex E, Cordeddu V et al. Diversity and functional consequences of germline and somatic PTPN11 mutations in human disease. Am J Hum Genet 2006;78:279-90. 
13. Tartaglia M, Mehler EL, Goldberg R, Zampino G, Brunner HG, Kremer $\mathrm{H}$ et al. Mutations in PTPN11, encoding the protein tyrosine phosphatase SHP-2, cause Noonan syndrome. Nat Genet 2001;29:465-8.

14. Tartaglia M, Niemeyer CM, Fragale A, Song X, Buechner J, Jung A et al. Somatic mutations in PTPN11 in juvenile myelomonocytic leukemia, myelodysplastic syndromes, and acute myeloid leukemia. Nat Genet 2003;34:148-50.

15. van der Burgt I, Berends E, Lommen E, van Beersum S, Hamel B, Mariman E. Clinical and molecular studies in a large Dutch family with Noonan syndrome. Am J Med Genet 1994;53:187-91.

16. Kotze LM. Coeliac disease. J Bras Gastroenterol 2006;6:23-34.

17. [No authors listed]. Revised criteria for diagnosis of coeliac disease. Report of Working Group of European Society of Paediatric Gastroenterology and Nutrition. Arch Dis Child 1990;65:909-11.

18. Barbieri D, Campos JV, Silva LM, Gonzales CH, Quarentei G, Brito T et al. A biópsia peroral do intestino delgado na criança. III. Resultados globais. Classificação dos padrões histológicos. Correlação estero-histológica. Síndrome pós-biópsia. Arq Gastroenterol 1970;7:141-50.

19. Hadithi M, de Boer H, Meijer JW, Willekens F, Kerckhaert JA, Heijmans R et al. Coeliac disease in Dutch patients with Hashimoto's thyroiditis and vice versa. World J Gastroenterol 2007;13:1715-22.

20. Rueda B, Koeleman BP, López-Nevot MA, Ortega E, Maldonado J, López M et al. Poly (ADP-ribose) polymerase-1 haplotypes are associated with coeliac disease. Int J Immunogenet 2005;32:245-8.
21. Watkins F, Fidler C, Boultwood J, Wainscoat JS. Mutations in PTPN11 are rare in adult myelodysplastic syndromes and acute myeloid leukemia. Am J Hematol 2004;76:417.

22. Salvia G, Cascioli CF, Ciccimara F, Terrin G, Cucchiara S. A case of proteinlosing enteropathy caused by intestinal lymphangiectasia in a preterm infant. Pediatrics 2001;107:416-7.

23. Lee WS, Boey CC, Goh AY, Chang KW, Iyngkaran N. Intestinal lymphangiectasia - a report of three chinese children in Malaysia. Singapore Med J 1998;39:418-21.

24. Nazer HM, Abultalib H, Hugosson C, al-Mahr M, Ali MA. Intestinal lymphangiectasia masquerading as coeliac disease. Ann Trop Paediatr 1991;11:349-55.

25. Aoyagi K, lida M, Yao T, Matsui T, Okada M, Oh K et al. Characteristic endoscopic features of intestinal lymphangiectasia: correlation with histological findings. Hepatogastroenterology 1997;44:133-8.

26. Yokocama M, Fagundes Neto U. Linfangiectasia Intestinal. The Electronic Journal of Pediatric Gastroenterology, Nutrition and Liver Diseases [serial on the internet]. 2006; 10(2) [cited 2009 Aug 25]. Available from: http://www.egastroped.com.br/sept03/linfangiectasia.htm

27. Ventura A, Magazzù G, Greco L. Duration of exposure to gluten and risk for autoimmune disorders in patients with celiac disease. SIGEP Study Group for Autoimmune Disorders in Celiac Disease. Gastroenterology 1999;117:297-303. 